\title{
Be different to be better: the effect of personality on optimal foraging with incomplete knowledge
}

\author{
Poppy M. Jeffries ${ }^{1} \mathbb{D} \cdot$ Samantha C. Patrick ${ }^{2}$. Jonathan R. Potts ${ }^{1}$
}

Received: 10 December 2020 / Accepted: 8 June 2021 / Published online: 25 June 2021

(c) The Author(s) 2021

\begin{abstract}
Many animal populations include a diversity of personalities, and these personalities are often linked to foraging strategy. However, it is not always clear why populations should evolve to have this diversity. Indeed, optimal foraging theory typically seeks out a single optimal strategy for individuals in a population. So why do we, in fact, see a variety of strategies existing in a single population? Here, we aim to provide insight into this conundrum by modelling the particular case of foraging seabirds, that forage on patchy prey. These seabirds have only partial knowledge of their environment: they do not know exactly where the next patch will emerge, but they may have some understanding of which locations are more likely to lead to patch emergence than others. Many existing optimal foraging studies assume either complete knowledge (e.g. Marginal Value Theorem) or no knowledge (e.g. Lévy Flight Hypothesis), but here we construct a new modelling approach which incorporates partial knowledge. In our model, different foraging strategies are favoured by different birds along the bold-shy personality continuum, so we can assess the optimality of a personality type. We show that it is optimal to be shy (resp. bold) when living in a population of bold (resp. shy) birds. This observation gives a plausible mechanism behind the emergence of diverse personalities. We also show that environmental degradation is likely to favour shyer birds and cause a decrease in diversity of personality over time.
\end{abstract}

Keywords Evolution $\cdot$ Optimal foraging theory $\cdot$ Personality $\cdot$ Seabird $\cdot$ Trade-off

\section{Introduction}

When should an animal leave its foraging patch in search of another? This is a core concern for understanding behavioural choices in animal populations, with a long history of theoretical and empirical study. In an environment where predators have complete knowledge of their surroundings, Charnov (1976) proposed that the optimal foraging strategy will depend on the quality and distribution of patches in the environment. Charnov's Marginal Value Theorem states that an animal should leave the patch it is foraging in when their rate of energy gain drops below the average energy

Poppy M. Jeffries

pmjeffries1@sheffield.ac.uk

1 School of Mathematics and Statistics, University of Sheffield, Hicks Building, Hounsfield Road, Sheffield S3 7RH, UK

2 School of Environmental Sciences, University of Liverpool, Nicholson Building, Brownlow Street, Liverpool L69 3GP, UK gain for the environment, which includes the cost of travelling between patches. Optimal foraging theory has been extended to more detailed stochastic models (Oaten 1977), which have been used to understand how model attributes affect foraging, such as prey distribution (Higginson and Ruxton 2015), patch distribution (Nonaka and Holme 2007; Rodríguez-Gironés and Santamaría 2006) and group competition (Laguë et al. 2012).

In the case of seabirds, however, individuals only have partial knowledge of their ocean environment, and are constantly gathering new information whilst foraging. Whilst predictability of foraging patches is low in oceanic waters, in temperate and polar regions, features such as continental shelf edges, frontal zones and upwellings create a slightly more predictable foraging habitat, which seabird populations have been observed to exploit (Weimerskirch 2007). The uncertainty in the environment and competition for more predictable resources creates an explorationexploitation trade-off foraging behaviour; the individual must chose to either explore the surrounding area to find higher quality resource patches and gain knowledge of 
their surroundings for future foraging trips, or exploit already-known habitats for food (Eliassen et al. 2007).

Recent studies have shown that an individual's choice of foraging strategy, i.e., where it lies on the exploration-exploitation trade-off, is highly correlated to its personality on the bold-shy continuum (Patrick et al. 2017). Bold birds tend to show higher levels of aggressive behaviour in response to novel objects, compared to shyer birds (Patrick et al. 2013). They are also more likely to favour exploration than their shyer counterparts; they will visit more patches, spending less time foraging in each and will spend less time searching between each patch. Shyer birds are more likely to favour exploitation; they will visit fewer patches, but they will fully exploit each patch they visit and will spend more time searching for higher quality patches (Patrick et al. 2017).

The aim of this paper is to explore seabird foraging strategies in an environment where individuals do not have complete knowledge of their surroundings. In particular, we focus on the differences in individual strategies that arise from personality differences along the shy-bold continuum within the population. In doing so, we aim to provide insight into why a diversity of personalities may have evolved, as well as understanding which strategies (and hence personalities) will be optimal in different environments. The ultimate aim is then to shed light on which personalities are likely to benefit or suffer from environmental change, and therefore give predictions as to how seabird personality is likely to evolve as a result of such changes.

For this, we propose a simple model to describe the energetics of seabirds foraging within a patchy resource environment. We assume that the location and quality of patches is not known to birds, but that they have some idea of the overall quality of the environment. In particular, we assume they have an idea of how long they are likely to need to search before finding their next patch. We begin by examining the optimal strategy for how long a bird should stay in a patch, assuming a fixed search time. The optimality question is phrased in two different ways: maximising rate of energetic gain and minimising the risk of unsuccessful foraging. The latter is defined to be a foraging trip where the energy lost through searching outweighs the energy gained through foraging.

We then examine the effect of personality on foraging strategy, by introducing a trade-off curve along the bold-shy continuum, whereby bold (resp. shy) birds have shorter (resp. longer) search times leading to discovery of the lower (resp. higher) quality patches. We calculate the optimal foraging strategy, and hence personality, given details about the personality make-up of the population. In doing so, we show whether it is optimal to be different or to be the same as everyone else.

\section{Single bird model}

We begin by considering the foraging behaviour of an individual seabird in the ocean, modelled as an environment with patchy resources. We examine individual in-patch foraging times for maximising rate of energy gain and minimising risk of energy loss.

Our model is split into two separate phases of behaviour: foraging in a patch and searching for a new patch. A seabird will forage in a patch. Then, when it is energetically favourable to, it will leave the patch to find a new patch. We define this process as a forage-search event, and each foraging trip will consist of multiple forage-search events. We determine how successful individuals are at foraging by considering their change in energy whilst foraging (Fig. 1).

During the foraging phase of the model, we consider the temporal behaviour of two variables: patch quality $p(t)$ and individual energy gain $u(t)$. Patch quality decays exponentially, whilst the individual gains energy from the patch at a rate proportional to the patch quality. The governing equations are

$\frac{d p}{d t}=-\gamma p$,

$\frac{d u}{d t}=\alpha p-\beta_{1}$,

with solutions

$p(t)=p_{0} e^{-\gamma t}$,

$u(t)=u_{*}+\frac{\alpha p_{0}}{\gamma}\left(1-e^{-\gamma t}\right)-\beta_{1} t$,

where $p_{0}>0$ is the initial patch quality, $\gamma>0$ is the rate of decay of the patch quality during foraging, $u_{*}>0$ is the initial energy of the individual upon entering the patch, $\alpha>0$ is the proportion of available forage converted to energy by an individual, and $\beta_{1}$ is the rate of energy loss due to metabolic functioning. Equation (3) assumes patch quality decays exponentially during foraging. Equation (4) assumes that the energy gain of an individual is proportional to the quality of the patch at time $t$, and that the individual loses energy due to metabolic functioning at a constant rate.

During the searching phase, we assume that an individual loses energy $u$ at an additional rate $\beta_{2}$ to account for additional energy costs whilst flying,

$u(t)=u_{*}-\left(\beta_{1}+\beta_{2}\right) t$,

where $u_{*}>0$ is the initial energy of the individual upon leaving the patch. We also model search times for an individual as a random variable $t_{s} \sim \operatorname{Exp}(\lambda)$. 
Fig. 1 An example of how energy is gained and lost by an individual during a foraging trip. During a foraging event, an individual gains energy at a rate proportional to the quality of patch. As the patch depletes, so too does the rate of energy gain, and at some point the bird decides to leave the patch in search of another

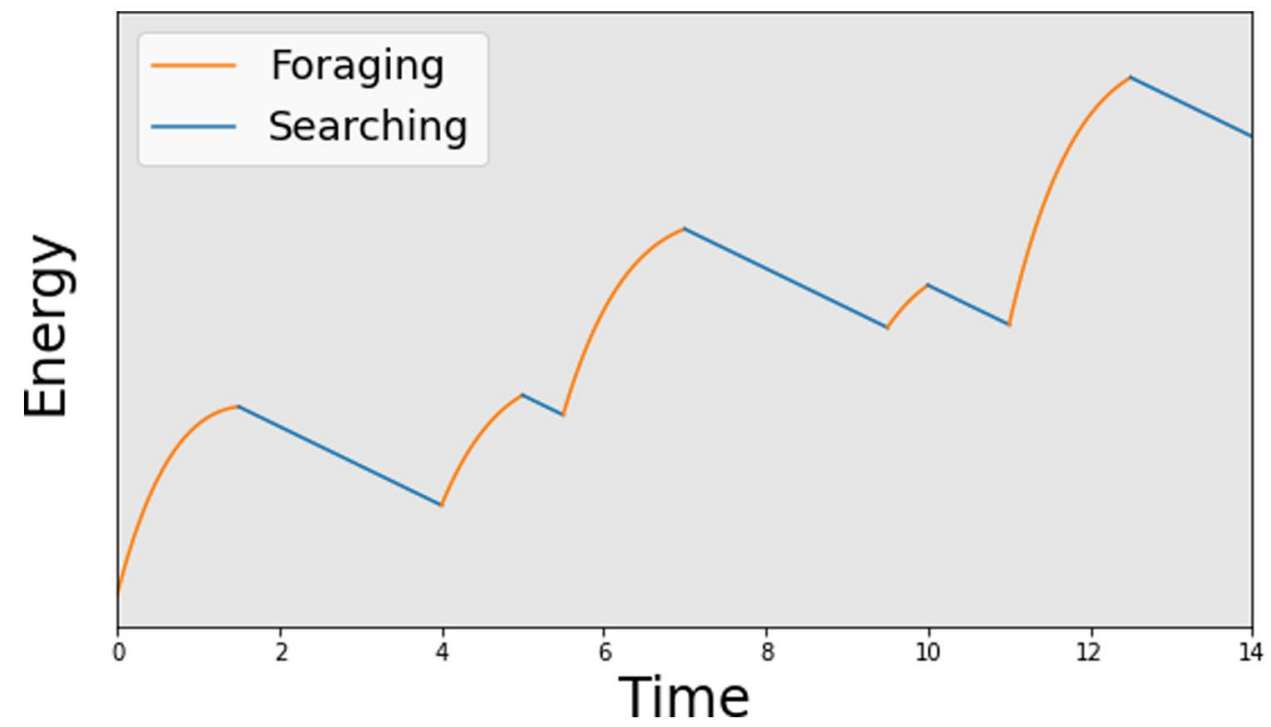

To analyse the behaviour of the model, we introduce dimensionless variables

$\tau=\gamma t, \quad \rho=\frac{\alpha p}{\beta_{1}}, \quad \mu=\frac{\gamma u}{\beta_{1}}$,

and dimensionless parameters into our model, as detailed in Table 1. In these dimensionless coordinates, the governing equations during the foraging phase are

$\frac{d \rho}{d \tau}=-\rho$,

$\frac{d \mu}{d \tau}=\rho-1$,

with solutions

$\rho(\tau)=\rho_{0} e^{-\tau}$,

$\mu(\tau)=\mu_{*}+\rho_{0}\left(1-e^{-\tau}\right)-\tau$,

whilst the governing equation for the searching phase is

$\frac{d \mu}{d \tau}=-(1+\beta)$

with solution

$\mu(\tau)=\mu_{*}-(1+\beta) \tau$.

\section{Foraging times}

When should an individual leave a patch in search of another? To forage successfully, an individual may want to attempt to maximise its rate of gain in energy. As foraging efficiency will decrease the longer an individual stays in a patch, the individual may wish to move to another patch soon after arriving at the first patch. However, due to uncertainty in knowledge of where patches will be and how high quality they will be, searching for a new patch can be risky.

Table 1 Glossary of model terms

\begin{tabular}{ll}
\hline & Definition \\
\hline$t$ & Time \\
$u(t)$ & Energy of an individual \\
$p(t)$ & Available forage in a resource patch \\
$\gamma$ & Resource patch decay rate during foraging \\
$p_{0}$ & Initial patch quality for a foraging phase \\
$\alpha$ & Proportion of available forage converted to energy by an \\
& individual \\
$\beta_{1}$ & Individual metabolic rate of energy loss \\
$\beta_{2}$ & Additional individual rate of energy loss whilst flying \\
& $\quad$ (searching) \\
$u_{*}$ & Initial individual energy for a foraging or searching phase \\
$t_{f}$ & Time spent foraging in a patch \\
$t_{s}$ & Time spent searching for a patch \\
$\lambda$ & Expected search rate, $t_{s} \sim$ Exp $(\lambda)$ \\
$\tau$ & Dimensionless variable $\tau=\gamma t$ \\
$\mu(\tau)$ & Dimensionless variable $\mu=\gamma u / \beta_{1}$ \\
$\rho(\tau)$ & Dimensionless variable $\rho=\alpha p / \beta_{1}$ \\
$\rho_{0}$ & Dimensionless parameter $\rho_{0}=\alpha p_{0} / \beta_{1}$ \\
$\beta$ & Dimensionless parameter $\beta=\beta_{2} / \beta_{1}$ \\
$\mu_{*}$ & Dimensionless parameter $\mu_{*}=\gamma u_{*} / \beta_{1}$ \\
$\tau_{f}$ & Dimensionless variable $\tau_{f}=\gamma t_{f}$ \\
$\tau_{s}$ & Dimensionless variable $\tau_{s}=\gamma t_{s}$ \\
$\mu_{f}$ & Dimensionless variable $\mu_{f}=\rho_{0}\left(1-e^{-\tau_{f}}\right)-\tau_{f}$ \\
$\mu_{s}$ & Dimensionless variable $\mu_{s}=(1+\beta) \tau_{s}$ \\
$\Lambda$ & Dimensionless parameter $\Lambda=\lambda / \gamma$ \\
\hline &
\end{tabular}


Longer search times may result in an individual losing more energy whilst searching than gained whilst foraging. This presents an alternative foraging strategy where an individual will stay foraging in a patch longer to minimise its risk of unsuccessful foraging; that is, losing more energy searching than gained foraging. In this section, we derive these optimal foraging times for both scenarios: maximising energy gain and minimising risk.

\section{Maximising rate of energy gain}

Given an expected search time, what is the optimal length of time for an individual to forage in a single patch in order to maximise its rate of energy gain? To answer this, we consider the rate of energy gain for one forage-search event. We consider the case where the search time is always $1 / \Lambda=\mathbb{E}\left[\tau_{s}\right]$, the mean of $\tau_{s}$, and assume the initial patch quality $\rho_{0}$ is known to the individual. The rate of energy gain for one forage-search event is given, in dimensionless parameters, by

$$
\begin{aligned}
\hat{r}\left(\tau_{f}\right) & =\frac{\mu_{f}-(1+\beta) \mathbb{E}\left[\tau_{s}\right]}{\tau_{f}+\mathbb{E}\left[\tau_{s}\right]} \\
& =\frac{\Lambda \rho_{0}\left(1-e^{-\tau_{f}}\right)-\Lambda \tau_{f}-(1+\beta)}{\Lambda \tau_{f}+1},
\end{aligned}
$$

where $\mu_{f}$ is the (dimensionless) energy gained whilst foraging, and $\tau_{f}$ and $\tau_{s}$ are the (dimensionless) times spent foraging and searching respectively (Table 1). Although a foraging trip will contain multiple forage-search events, we base our analysis on one and assume that patch quality does not vary sufficiently to have a big effect on optimality.
The rate of energy gain $\hat{r}$ is maximised when $\tau_{f}=\tau_{f}^{*}$, given by

$\tau_{f}^{*}=-W\left(\frac{\left(\beta-\Lambda \rho_{0}\right) e^{-(1+\Lambda) / \Lambda}}{\Lambda \rho_{0}}\right)-\frac{1+\Lambda}{\Lambda}$,

where $W$ is the Lambert $W$ function, the inverse of the function $f(z)=z e^{z}$ (Appendix 7). In Fig. 2, we see that this time increases as expected search time increases, initial patch quality decreases, and the flight energy cost increases. All of these three situations can be considered as examples of worsening environmental conditions for the animal.

\section{Minimising the risk of unsuccessful foraging}

The environment which seabirds forage in is uncertain, and changing environmental conditions will likely increase this uncertainty. Environmental uncertainty motivates an individual foraging time which aims to minimise the probability that a forage-search event is unsuccessful. Here we revert to modelling search times as a random variable, $\tau_{s} \sim \operatorname{Exp}(\Lambda)$, and ask how long should an individual forage in a patch to minimise their risk of losing energy during a forage-search event?

To answer this, we calculate the probability, $\mathrm{P}\left(\mu_{s}>\mu_{f}\right)$, that one forage-search event is unsuccessful, i.e. the (dimensionless) energy gained whilst foraging, $\mu_{f}$, is less than the (dimensionless) energy lost whilst searching, $\mu_{s}$. As $\mu_{s}$ is dependent on the random variable $\tau_{s}$ (Table 1 ), we have that

$$
\begin{aligned}
\mathrm{P}\left(\mu_{s}>\mu_{f}\right) & =\exp \left(-\frac{\Lambda \mu_{f}}{1+\beta}\right) \\
& =\exp \left(-\frac{\Lambda}{1+\beta}\left[\rho_{0}\left(1-e^{-\tau_{f}}\right)-\tau_{f}\right]\right) .
\end{aligned}
$$
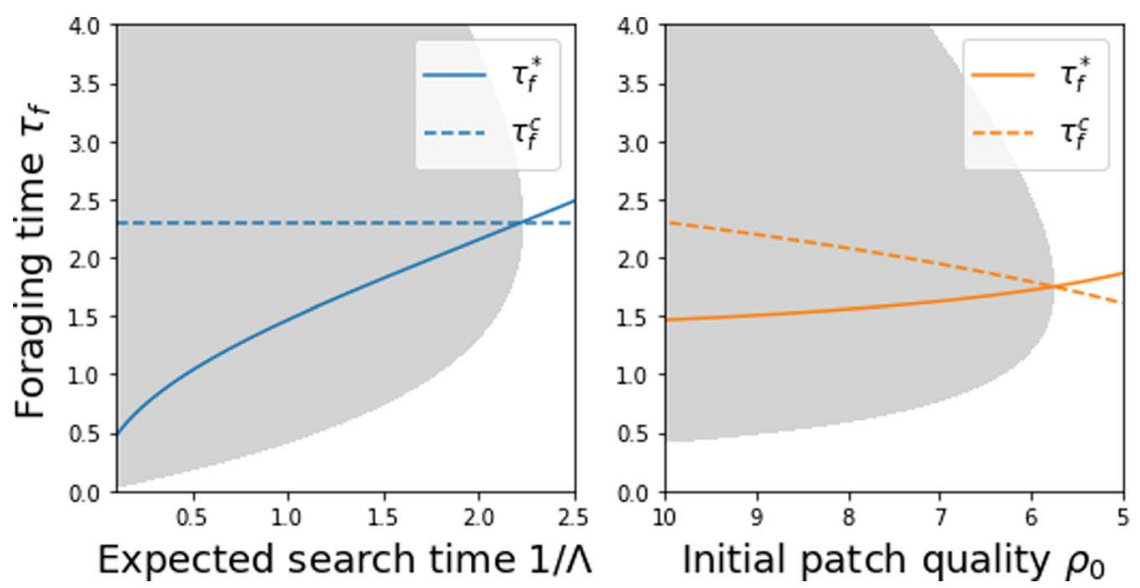

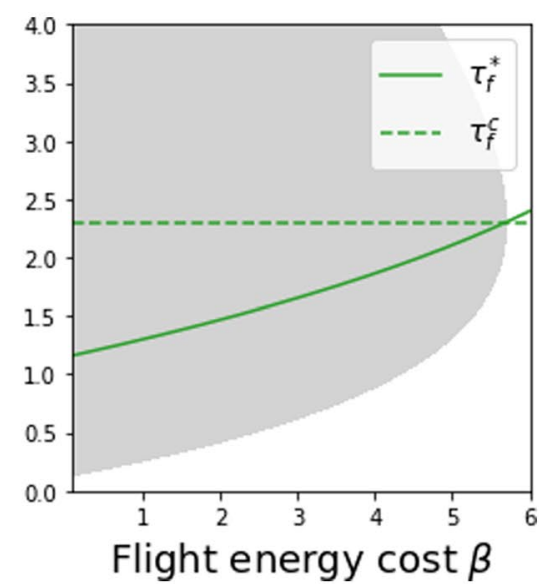

Fig. 2 The effect of increasing the expected search time, decreasing initial patch quality and increasing flight energy cost on the optimal foraging time, $\tau_{f}^{*}$, that maximises net energetic gain, as in Eq. (14), and the minimal risk foraging time, $\tau_{f}^{c}$, that minimises risk, as in Eq.
(16). Shaded areas show where foraging is successful, i.e. $\hat{r}\left(\tau_{f}\right)>0$ as described in Eq. (13). When fixed, $\beta=2, \Lambda=1$ and $\rho_{0}=10$. Note the $\rho_{0}$ axis is reversed 
This probability is minimised by $\tau_{f}=\tau_{f}^{c}$, where

$\tau_{f}^{c}=\ln \rho_{0}$.

This foraging time maximises the amount of energy gained during the foraging phase, $\mu_{f}$. Therefore, to minimise the probability of an unsuccessful foraging event, the individual should stay foraging in the patch until their rate of net energy gain becomes negative. As $\tau_{f}^{c}$ only depends upon $\rho_{0}$, this minimum risk foraging time is only affected by the patch quality, $p_{0}$ and the individual's foraging ability, quantified by $\alpha$, the rate at which available forage is converted to energy (Table 1). (Note that parameters $\beta$ and $\Lambda$ only affect the probability of an unsuccessful forage-search event, and not the time an individual should stay in a patch to minimise this probability.)

\section{Analysis of foraging times}

In Fig. 2 we compare the effect of model parameters on the foraging time that maximises the rate of energy gain (Eq. 14), and the foraging time that minimises the probability of net energy loss (Eq. 16). Increasing dimensionless parameter $1 / \Lambda$ increases the expected search time $1 / \lambda$, decreasing dimensionless parameter $\rho_{0}$ decreases the initial patch quality $p_{0}$, and increasing dimensionless parameter $\beta$ increases the flight energy cost $\beta_{1}$. The time $\tau_{f}^{*}$ increases as the search times get longer, the patch quality decreases, and the flight energy cost increases. All of these factors can be considered as features that make the environment less favourable for foraging seabirds. Consequently, the rule of thumb is that, to maximise energetic gain, a poorer environment for foraging should be mitigated by longer foraging bouts.

The minimal risk foraging time, $\tau_{f}^{c}$, however, shows a rather different trend. This value only depends on $\rho_{0}$. Converse to the effect of $\rho_{0}$ on $\tau_{f}^{*}$, here we see that $\tau_{f}^{c}$ decreases as $\rho_{0}$ decreases. This means that, as conditions worsen, individuals should decrease foraging time if they are seeking to reduce risk, but increase foraging time if seeking to maximise average gains. Note that as conditions worsen and the rate of energy gain $\hat{r}\left(\tau_{f}\right)$ decreases to 0 , the optimal foraging times under our two different optimality scenarios, $\tau_{f}^{*}$ and $\tau_{f}^{c}$, converge until $\tau_{f}^{*}=\tau_{f}^{c}$, when $\hat{r}\left(\tau_{f}\right)=0$. When $\hat{r}\left(\tau_{f}\right)=0$, the energy gained foraging is equal to the energy lost whilst searching. In the scenario where the optimal rate of energy gain an individual can achieve is 0 , the associated optimal foraging time will be the time taken to maximise energy gain whilst in a patch, else the individual would be able to adjust their foraging time to achieve a positive rate of energy gain. This foraging time would be $\tau_{f}^{*}=\ln \rho_{0}$, which is equivalent to $\tau_{f}^{c}$.
In Fig. 3a we compare the effect of model parameters on the optimal rate of energy gain $\hat{r}\left(\tau_{f}^{*}\right)$ (Eq. 13) when $\tau_{f}=\tau_{f}^{*}$ (Eq. 14). In Fig. 3b we compare the effect of model parameters on $P\left(\mu_{s}>\mu_{f}^{c}\right)$, the minimal risk of an unsuccessful forage-search event (Eq. 15) when $\tau_{f}=\tau_{f}^{c}$ (Eq. 16). We see the optimal rate of energy gain decreases and the probability of risk increases under less favourable conditions: longer expected search times, higher flight energy costs, and lower initial patch qualities.

\section{Personality as a driver for a foraging strategy trade-off}

We have presented two indicators of foraging success for individuals choosing the length of time they forage in a patch: optimising the rate of energy gain and minimising the risk of unsuccessful foraging. Whilst foraging time is the only parameter individuals have complete control over, individuals can prioritise patches of higher quality or shorter search times between patches. This results in a foraging strategy trade-off.

Different individual personalities tend to prefer different foraging strategies (Patrick and Weimerskirch 2014; Patrick et al. 2017). Bolder personalities favour exploration; they will move quickly around the environment (high expected search rate $\Lambda$ and short expected search time $1 / \Lambda$ ), visiting more patches which are more likely to be smaller and of lower quality (low initial patch quality $\rho_{0}$ ). Shyer personalities favour exploitation; they will spend longer searching for a patch (low $\Lambda$, short $1 / \Lambda$ ), visiting fewer patches of higher quality (high $\rho_{0}$ ).

\section{Trade-off}

We model this foraging strategy trade-off by extending our forage-search model presented in Eqs. (3)-(5) to include a trade-off between $\rho_{0}$, the initial patch quality, and $\Lambda$, the expected search rate. We use the following (phenomenological) functional form for our trade-off curve

$\rho_{0}=c_{1}\left(\frac{c_{2}}{\Lambda}\right)^{b}+1$,

where $c_{1}$ and $c_{2}$ are constants. The +1 term enforces the $\rho_{0}>1$ condition required to make the rate of gain in energy whilst foraging in Eq. (8) initially positive. The shape of the trade-off curve is modified to account for competition between individuals with a similar strategy by varying $b$, a measure of population boldness. When $b$ is increased, bold individuals have lower patch qualities and shy individuals 


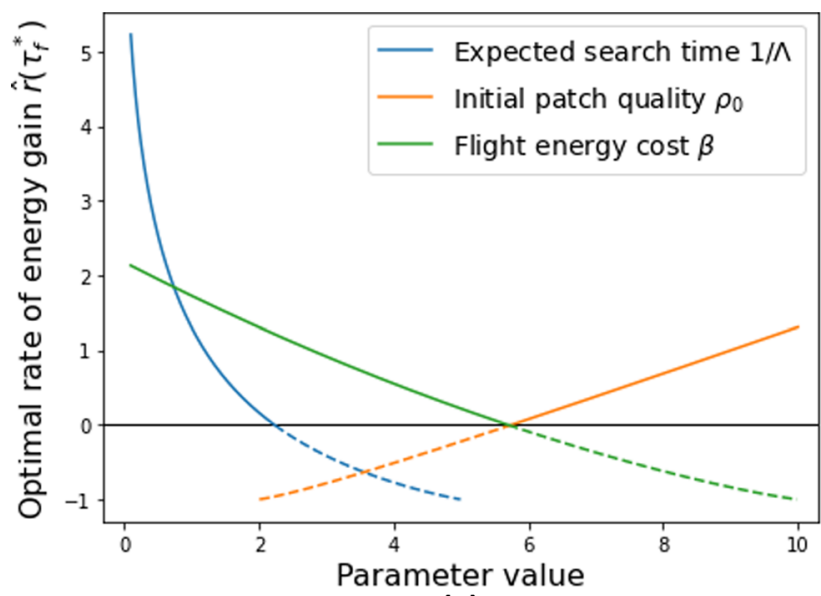

(a)

Fig. 3 The effect of varying the expected search time, initial patch quality and flight energy cost on (a) the optimal rate of energy gain $\hat{r}\left(\tau_{f}^{*}\right)$ given by Eq. (13) when $\tau_{f}=\tau_{f}^{*}$ as in Eq. (14), and (b) the probability of an unsuccessful forage-search event given by Eq. (15), for the minimal risk foraging time when $\tau_{f}=\tau_{f}^{c}$, as in Eq. (16). When

have higher patch qualities to account for a change in competition, and vice-versa as $b$ is decreased.

Figure 4 shows example trade-off curves. Individual foraging strategies are represented by a point on a given trade-off curve, which dictates the expected search time and patch quality during a forage-search event. We assume that $\rho_{0}$ is the quality of a typical foraging patch for an individual with expected search time $1 / \Lambda$. Whilst initial patch quality will vary across multiple forage search events, and these initial patch qualities will be unknown to the individual until they reach a patch, we assume $\rho_{0}$ is a mean and variation around this mean will not have a large effect on optimality. There is an intermediate strategy at $\Lambda=c_{2}$ and $\rho_{0}=c_{1}+1$. Bolder individuals with shorter expected search times and lower patch qualities lie on the curve where $\Lambda>c_{2}$ and $\rho_{0}>c_{1}+1$, whilst shy individuals with higher patch qualities and longer expected search times lie on the curve where $\Lambda<c_{2}$ and $\rho_{0}<c_{1}+1$. As we vary population boldness $b$, we assume the expected search time for each individual, $1 / \Lambda$, remains fixed. Increasing population boldness $b$ results in bold individuals with higher $\Lambda$, having a lower initial patch quality $\rho_{0}$ due to increased competition, and shy individuals with a lower $\Lambda$, having a higher initial patch quality $\rho_{0}$ due to decreased competition.

\section{Optimal rate analysis}

We measure foraging success by the rate of energy gain $\hat{r}\left(\tau_{f}\right)$ during a forage-search event, as defined in Eq. (13). When evaluating $\hat{r}$ at $\tau_{f}=\tau_{f}^{*}$, the optimal foraging time in Eq. (14),

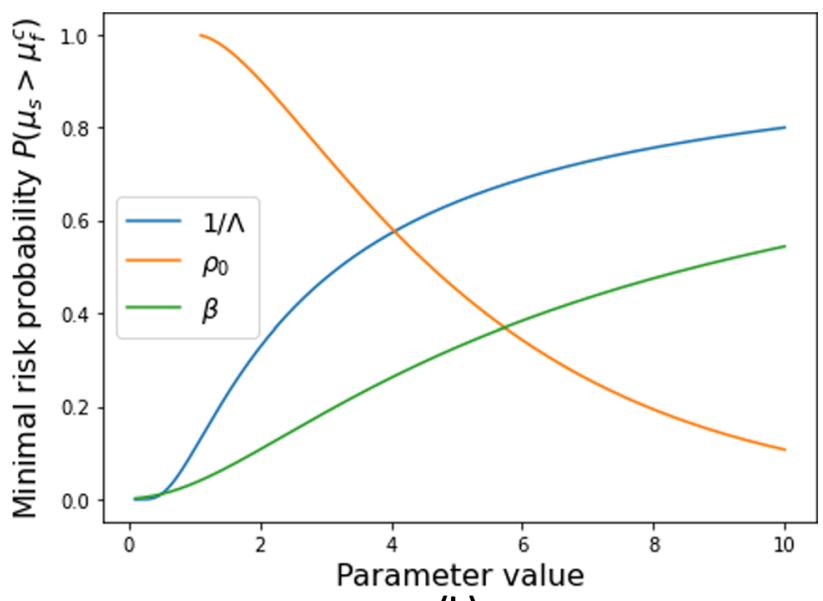

(b)

fixed, $\beta=2, \Lambda=1$ and $\rho_{0}=10$. Solid lines represent when rate of energy gain is positive, $\hat{r}\left(\tau_{f}^{*}\right)>0$. Dashed lines represent when $\hat{r}\left(\tau_{f}^{*}\right)<0$; the individual has been unsuccessful at having a net energy gain during a forage-search event

we obtain the optimal rate of energy gain $\hat{r}\left(\tau_{f}^{*}\right)$ for an individual with expected search time $1 / \Lambda$. How does population boldness affect this? Substituting in the foraging strategy trade-off in Eq. (17) gives

$\hat{r}\left(\tau_{f}^{*}\right)=\frac{\Lambda\left(c_{1} c_{2}^{b} \Lambda^{-b}+1\right)\left(1-e^{-\tau_{f}^{*}}\right)-\Lambda \tau_{f}^{*}-(1+\beta)}{\Lambda \tau_{f}^{*}+1}$,

where

$\tau_{f}^{*}=-W\left(\frac{\left(\beta-\Lambda\left(c_{1} c_{2}^{b} \Lambda^{-b}+1\right)\right) e^{-(1+\Lambda) / \Lambda}}{\Lambda\left(c_{1} c_{2}^{b} \Lambda^{-b}+1\right)}\right)-\frac{1+\Lambda}{\Lambda}$,

is the optimal foraging time for an individual of expected search time $1 / \Lambda$ within a population of boldness $b$. In Fig. 5 we see that this foraging time increases for individuals with longer expected search times (i.e. shyer individuals).

Maximising $\hat{r}\left(\tau_{f}^{*}\right)$ with respect to $\Lambda$ gives the optimal position on the trade-off curve, with expected search time denoted by $1 / \Lambda^{*}$ (derived in Appendix 8 ). This search time gives the most successful individual foraging strategy for an individual foraging in a population of boldness $b$, as presented in Fig. 6. As population boldness increases, the optimal expected search time $1 / \Lambda^{*}$ increases, so in a population of bolder individuals, individuals with a longer expected search time (i.e. shyer individuals) are most successful, and vice-versa.

We also look at the success of the population as a whole, by considering $b$-values that represent a shy population, an intermediate population, and a bold population (insets of 
Fig. 4 Proposed $\Lambda-\rho_{0}$ tradeoff as in Eq. (17) with $c_{1}=4$, $c_{2}=4$ and $b \approx 0.45$ for the intermediate population, $b=0.3$ for a shyer population and $b=0.6$ for a bolder population

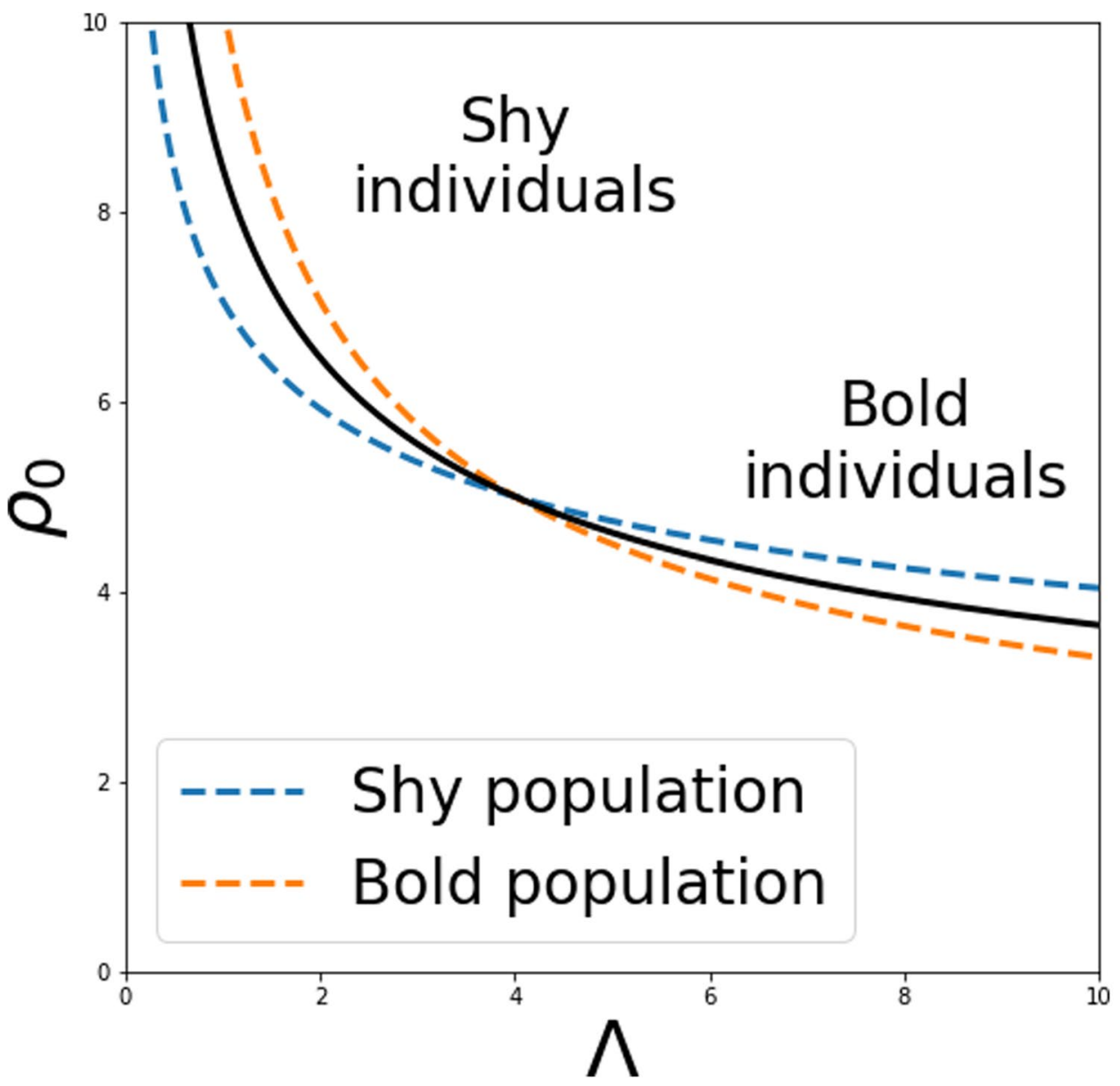

Fig. 6). The intermediate population is defined as the $b$-value for which the intermediate individual strategy is

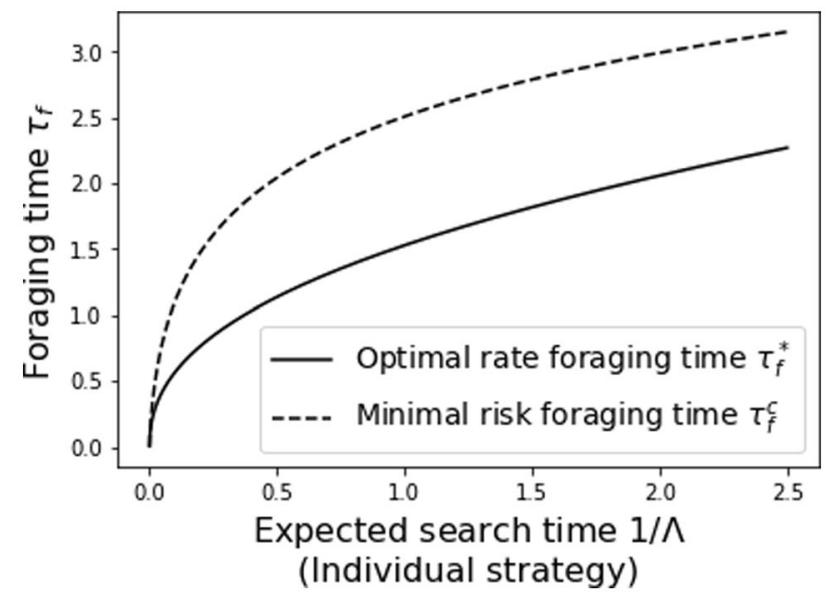

Fig. 5 The optimal rate foraging time (Eq. 19), and the minimal risk foraging time (Eq. 20) for individuals of expected search times $1 / \Lambda$. Shy individuals have longer expected search times, and bold individuals have shorter expected search times, as described by the trade-off curve (Eq. 17) and shown in Fig. 4. Model parameters are $\beta=2$, $c_{1}=4$ and $c_{2}=4$. Population boldness $b$ is shown as the intermediate population boldness for each analysis: $b \approx 0.45$ for $\tau_{f}^{*}$ (derived in Appendix 8.1) and $b \approx 0.75$ for $\tau_{f}^{c}$ (derived in Appendix 10.1) the optimal expected search time, $1 / \Lambda^{*}=1 / c_{2}$ (derived in Appendix 8.1). In both the bold and shy populations, we see the individuals with highest optimal rates of energy gain $\hat{r}\left(\tau_{f}^{*}\right)$ are those with contrasting personalities to the rest of their population. In the intermediate population, the optimal expected search time $1 / \Lambda^{*}$ has a lower optimal rate of energy gain $\hat{r}\left(\tau_{f}^{*}\right)$ than in the bold and shy population. Individuals with expected search times close to the intermediate strategy are more successful than individuals with outlying strategies.

\section{Minimal risk analysis}

Here, we measure the risk of unsuccessful foraging by considering the probability, $P\left(\mu_{s}>\mu_{f}\right)$, that the net energy change in one forage-search event is negative. How does population boldness affect an individual's risk of unsuccessful foraging? Recall that this risk is minimised by $\tau_{f}=\tau_{f}^{c}$ (Eq. 16). Substituting the trade-off curve from Eq. (17) into Eq. (16), we see that the minimal risk foraging time for an individual with expected search time $1 / \Lambda$ in a population of boldness $b$ is 


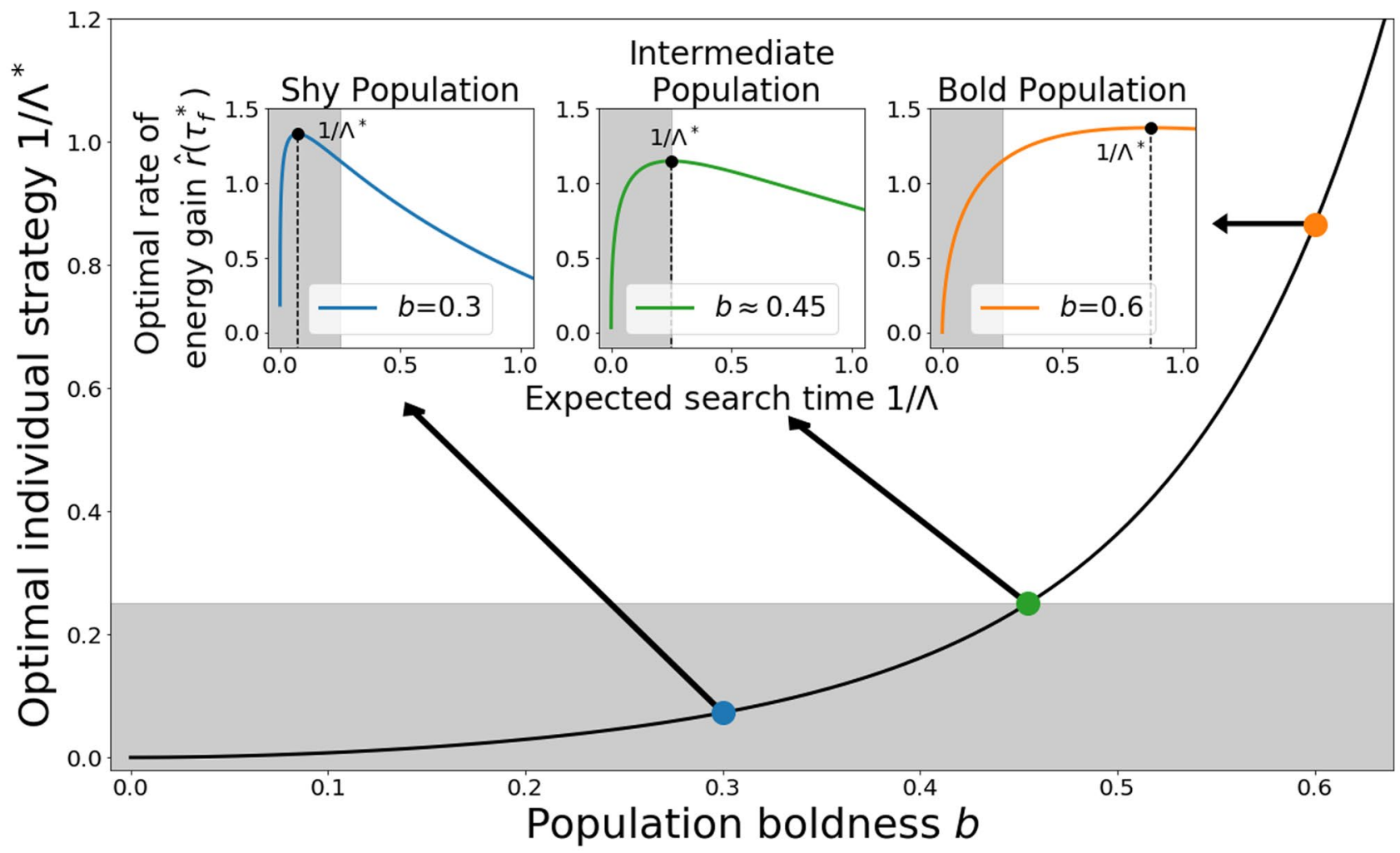

Fig. 6 Insets: The optimal rate of energy gain $\hat{r}\left(\tau_{f}^{*}\right)$, as defined in Eq. (18), for individuals of expected search times $1 / \Lambda$, in various populations of boldness $b$. Main: The optimal individual expected search time $1 / \Lambda^{*}$ in a population of boldness $b$, given by the individual strat-

$\tau_{f}^{c}=\ln \left(c_{1} c_{2}^{b} \Lambda^{-b}+1\right)$.

In Fig. 5 we see that this foraging time increases for individuals with longer expected search times (shyer individuals). The probability of foraging unsuccessfully given this minimal risk foraging time is then

$\mathrm{P}\left(\mu_{s}>\mu_{f}^{c}\right)=\left(\left(c_{1} c_{2}^{b} \Lambda^{-b}+1\right) e^{-\left(c_{1} c_{2}^{b} \Lambda^{-b}\right)}\right)^{\Lambda /(1+\beta)}$.

By minimising Eq. (21) with respect to $\Lambda$, we find the search time required to minimise the probability of unsuccessful foraging. This is given implicitly by the following equation (see Appendix 10 for derivation)

$$
\begin{aligned}
& (b-1)\left(c_{1} c_{2}^{b} \Lambda^{-b}\right)^{2}-c_{1} c_{2}^{b} \Lambda^{-b} \\
& +\left(c_{1} c_{2}^{b} \Lambda^{-b}+1\right) \ln \left(c_{1} c_{2}^{b} \Lambda^{-b}+1\right)=0 .
\end{aligned}
$$

We denote by $\Lambda_{c}(b)$ the value of $\Lambda$ that solves Eq. (22) for a given value of $b$.

Figure 7 shows the relationship between population boldness $b$ and the expected search time $1 / \Lambda_{c}$ of egy with the maximal optimal rate of energy gain $\hat{r}\left(\tau_{f}^{*}\right)$, as defined in Eq. (18). Shaded areas show individuals with expected search times that are lower than that of the intermediate individual (i.e. bolder individuals). Model parameters are $\beta=2, c_{1}=4$ and $c_{2}=4$

an individual choosing an optimal strategy for minimising risk. In a population of bolder individuals, it is optimal to be a shy individual with a longer expected search time, and in a population of shyer individuals, it is optimal to be a bold individual with a shorter expected search time.

We also look at the minimal individual risk within the whole population, for selected $b$-values showing a shy, intermediate, and bold population (insets of Fig. 7). Again the intermediate population is defined as the $b$ value for which the minimal risk individual strategy is the intermediate expected search time, $1 / \Lambda^{*}=1 / c_{2}$ (derived in Appendix 10.1). In a population of shyer individuals, the individuals with lowest individual minimal risk are relatively bold, and in a population of bolder individuals, the individuals with lowest risk are relatively shy. In the intermediate population, the minimal risk individual strategy $1 / \Lambda_{c}$ has higher risk than in the bold and shy population. Individuals with expected search times close to the intermediate strategy have lower risk than individuals with outlying strategies. 


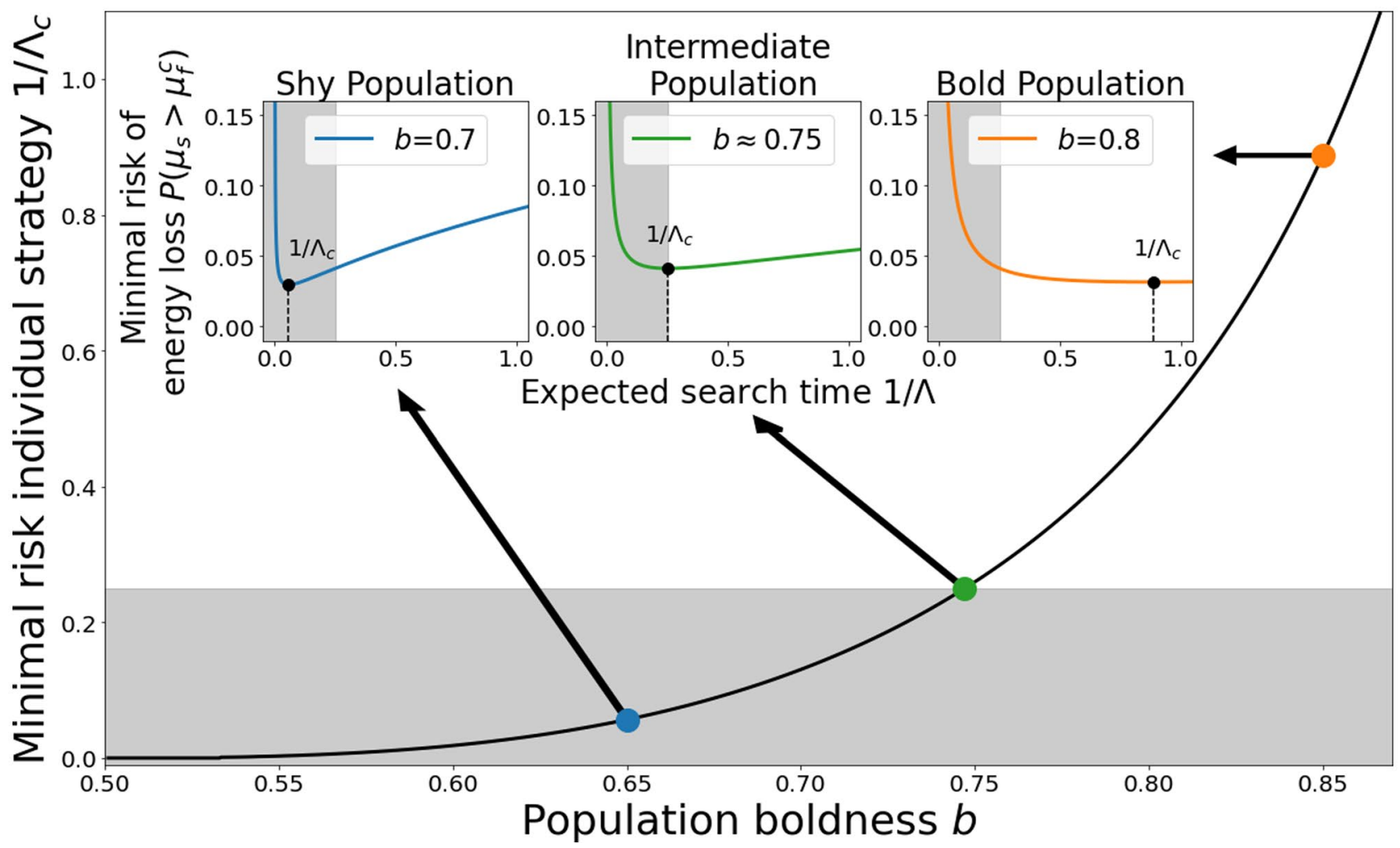

Fig. 7 Insets: The minimal risk of energy loss $P\left(\mu_{s}>\mu_{f}^{c}\right)$, as defined in Eq. (21), for individuals with expected search times $1 / \Lambda$, in various populations of boldness $b$. Main: The minimal risk individual strategy $1 / \Lambda_{c}$ in a population of boldness $b$, given by the individual

\section{Discussion and conclusions}

We have derived analytic expressions for optimal foraging strategies in a patchy environment on which individuals only have partial knowledge. We have shown how these strategies depend upon the underlying environment, the individual's personality, and the personality composition of the population. Previous empirical work has shown that personality affects an individual's foraging strategy along the exploration-exploitation trade-off (Patrick et al. 2017) but it is not fully resolved as to why different strategies should exist in different individuals. By modelling an individual's personality as a position on this trade-off curve, we have shown that it is optimal to have a different personality to the rest of the population. Specifically, in a population of shy (resp. bold) individuals, it is optimal to be bold (resp. shy) (Figs. 6 and 7). From this, we expect that over time shy (resp. bold) populations would become more bold (resp. shy), moving towards an intermediate population. In principle, an intermediate population would remain homogeneous. However, it is likely that bolder or shyer individuals would enter the population through birth or immigration, creating a need for the population to be rebalanced by shyer or bolder strategy with the minimal probability of energy loss $P\left(\mu_{s}>\mu_{f}^{c}\right)$, as defined in Eq. (21). Shaded areas show individuals with expected search times that are lower than that of the intermediate individual (i.e. bolder individuals). Model parameters $\beta=2, c_{1}=4$ and $c_{2}=4$

individuals. This supports evidence showing that personality may be mainted in the population due to frequency dependent selection (FDS) linked to reproduction (Dingemanse et al. 2004) and life history trade-offs (Wolf et al. 2007).

Our conclusions can be viewed as an example of negative FDS. FDS is known to act throughout the animal kingdom as rarity minimises competition and conspecific encounter. Studies have shown the FDS can give rise to, and maintain, personality difference themselves (e.g. Wolf and McNamara 2012) and foraging specialisations (e.g. Bolnick et al. 2003; Rueffler et al. 2006). Our study builds on this work and shows that FDS alters the optimal exploration-exploitation strategy in a population. Assuming personality is fixed within an individual (Sih et al. 2004), selection on the strategy individuals use to acquire resources may lead to selection on personality type itself, and alter the composition of populations.

To assess the foraging success of individuals, we present two theoretical optimal foraging times for individuals foraging in a patchy ocean environment: optimising the net rate of energy gain (the optimal rate strategy), and minimising the risk of losing more energy searching for a new patch than gained foraging (the minimal risk strategy). Optimal foraging theory (Charnov 1976) presents an optimal time 
for foraging in a known environment, via the Marginal Value Theorem (MVT). Indeed, if one sets $\beta=0$ (no energetic cost of movement) in our single-individual optimal rate strategy model, we have the same scenario as in MVT and return the same optimal foraging time, given in Eq. (14).

However, seabirds do not have such complete, deterministic knowledge so are unable to determine this optimal time. Rather, individuals will have knowledge of the patch they are currently foraging in, as well as an intrinsic strategy (along the bold-shy continuum) which determines the amount of time they are likely to spend searching for a good patch and consequently the quality of the patches they are likely to settle in. This leads to both our examination of the minimum risk strategy as well as the effect of personality on optimality. Our study can be viewed as providing a general theory of optimal foraging whenever such partial environmental knowledge and personality variations are in place.

For an individual to minimise its probability of foraging unsuccessfully, it should stay in a patch until it is fully exploited (Eq. 16); that is, when the rate of energy gain from foraging drops below the rate of metabolic energy loss. However, for an individual to maximise its rate of energy gain, it should leave earlier (Eq. 14), even though the rate of energy gain from the patch is higher than the rate of metabolic energy loss. Less favourable conditions (i.e. longer expected search times, lower initial patch qualities and increased flight energy cost) lead to an increase in optimal rate foraging time, which converges to the minimal risk foraging time as conditions become so bad that the foraging environment becomes unprofitable (Fig. 2). Less favourable conditions also lead to individuals being less successful at gaining energy whilst foraging, and incurring an increased risk of foraging unsuccessfully (Fig. 3).

As well as modelling a single individual, we also compared foraging times and success of different personalities within a population, via the inclusion of the foraging strategy trade-off (Eq. 17, Fig. 4). We showed that it is optimal for shy individuals, who prioritise higher patch qualities over shorter search times, to stay in patches longer to maximise their net energy gain whilst foraging (Fig. 5), which supports empirical observations in seabirds (Patrick et al. 2017). In less favourable environments, the optimal rate foraging time increases; therefore shy birds will be more resilient to the effects of a degrading environment. Conversely, bolder birds may have increased foraging success if climate change causes their environment to improve locally.

Our modelling framework is based on the concept of energetic costs and gains. These vary depending on both the species being studied, due to differences in physical features such as body size and wing span, and environmental conditions such as wind. A key parameter in our model is the effect of flight energy costs on individual foraging success, given by the ratio $\beta=\beta_{2} / \beta_{1}$ (Table 1 ), where $\beta_{1}$ is the metabolic energy rate, and $\beta_{2}$ is the flight energy cost (Dunn et al. 2018). Calculating this ratio for a particular species requires careful consideration of physical and environmental factors. For example, wandering albatross use increased winds to their advantage, saving energy costs by soaring, whereas diving birds such as puffins have high energy costs fighting against the wind (Elliott et al. 2014; Weimerskirch et al. 2000, Furness and Bryant 1996). Kittiwakes likewise have relatively high flight energy costs; they can soar but high winds increase flapping time (Gabrielsen et al. 1987).

The optimal rate of energy gain decreases with $\beta$ and the risk of unsuccessful foraging increases (Figs. 2 and 3). Consequently, birds that have increased flight energy cost (e.g. due to being less efficient at flying long distances) have a higher probability of the forage-search event being unsuccessful. In this case, it is more optimal to spend a long time depleting a patch before moving on (a conservative strategy). Conversely, birds who are able to soar for long distances without expending much energy may benefit by moving on from a patch before it has been depleted in order to search for a better patch (a less conservative strategy).

As well as seabird physiology, the overall quality of the environment is also a key aspect of our model. This comes in via the parameter $\rho_{0}$ (Table 1) which can be thought of as a dimensionless proxy for the quality of a typical foraging patch. Both increased water temperatures and the presence of fisheries can have an effect on patch quality (Furness and Tasker 2000). Anthropogenic actions are responsible for current changes in both these factors. We have shown that these can result in changes in optimal behaviour (Fig. 3) and therefore alter the evolutionary trajectory of seabirds.

Alongside changes in overall environmental quality, changes in environmental uncertainty can also affect the optimal foraging strategy. Such uncertainty is likely to lead to longer expected search times, which can be viewed through the dimensionless parameter $1 / \Lambda$ in our model. As $1 / \Lambda$ increases, the rate energy gain decreases and the probability of an unsuccessful forage increases (Fig. 3). Consequently, similar to environmental degradation, environmental uncertainly can have a negative effect on foraging and may drive changes in the personality composition of seabird populations towards favouring a narrow and shyer range of personalities. We are currently examining an individual based model that builds on this to incorporate explicitly the effects of movement, variable patch quality, and competition for resources, in which we aim to gain more detailed insight into the effects of environmental changes on the evolution of personality, and will be the subject of future work.

In conclusion, we have given mechanistic underpinnings for the emergence of differing personalities in seabird populations, via consideration of their foraging strategies in environments on which they have partial knowledge. Furthermore, we have shown that changes in environment, such as those currently caused by anthropogenic actions (e.g. increased fishing, climate 
change), will affect the optimal personality types and thus the evolution of personality in seabird populations. Overall, we predict that environmental degradation, either by increased uncertainty or decreased overall foraging quality, will cause the personality pool of seabirds to become shyer and less diverse.

\section{Appendix}

\section{Optimal foraging time: Lambert $\boldsymbol{W}$ function}

The Lambert $W$ function is multivalued, and for $\tau_{f}^{*}$ (Eq. 14) to be a maximum we require the lower branch, denoted $W_{-1}(z)$, with $-e^{-1} \leq z<0$ and $W_{-1}(z) \leq-1$. As we require $z<0$, from Eq. (14) we deduce that

$\beta<\Lambda \rho_{0}$,

for real solutions. When the condition is not met, the maximum does not exist and $\hat{r} \rightarrow-1$ as $\tau_{f} \rightarrow \infty$. Hence when $\beta \geq \Lambda \rho_{0}$ the conditions on the model are so unfavourable that the most successful strategy is to stay foraging in the same patch, even as the rate of energy gain becomes negative, as searching is too costly or risky. This is illustrated in Fig. 2 as increasing $\beta$ or decreasing $\Lambda$ eventually results in the optimal foraging time tending to infinity. We also see that as $\rho_{0}$ decreases to $1, \tau_{f}^{*} \rightarrow \infty$; as the quality of patches decreases, the reward of searching for a new patch becomes too low to make searching worthwhile.

\section{Derivation of optimal rate individual in a population}

We define

$z=\frac{\left(\beta-\Lambda\left(c_{1} c_{2}^{b} \Lambda^{-b}+1\right)\right) e^{-(1+\Lambda) / \Lambda}}{\Lambda\left(c_{1} c_{2}^{b} \Lambda^{-b}+1\right)}$.

such that the optimal foraging time in Eq. (19) becomes

$\tau_{f}^{*}=-W(z)-\frac{1+\Lambda}{\Lambda}$.

Then differentiating the optimal rate of energy gain for an individual, given in Eq. (18), with respect to $\Lambda$ gives

$$
\begin{aligned}
\frac{d \hat{r}\left(\tau_{f}^{*}\right)}{d \Lambda}= & \frac{\left(\rho_{0}+\Lambda \rho_{0}^{\prime}\right)\left(1-e^{-\tau_{f}^{*}}\right)+\Lambda \rho_{0} \tau_{f}^{*^{\prime}} e^{-\tau_{f}^{*}}-\tau_{f}^{*}-\Lambda \tau_{f}^{*^{\prime}}}{\Lambda \tau_{f}^{*}+1} \\
& -\frac{\left(\tau_{f}^{*}+\Lambda \tau_{f}^{*^{\prime}}\right)\left(\Lambda \rho_{0}\left(1-e^{-\tau_{f}^{*}}\right)-\Lambda \tau_{f}^{*}-(1+\beta)\right)}{\left(\Lambda \tau_{f}^{*}+1\right)^{2}},
\end{aligned}
$$

where $\rho_{0}$ is described by the foraging strategy trade-off in Eq. (17) and

$\rho_{0}^{\prime}=\frac{d \rho_{0}}{d \Lambda}=-b c_{1} c_{2}^{b} \Lambda^{-(b+1)}$,

$$
\begin{aligned}
\tau_{f}^{*^{\prime}}=\frac{d \tau_{f}^{*}}{d \Lambda} & =-z^{\prime}(W(z))^{\prime}+\frac{1}{\Lambda^{2}} \\
& =-\frac{z^{\prime} W(z)}{z(1+W(z))}+\frac{1}{\Lambda^{2}},
\end{aligned}
$$

$$
\begin{aligned}
& z^{\prime}=\frac{d z}{d \Lambda}= \frac{\left(\beta-\Lambda \rho_{0}-\Lambda^{2} \rho_{0}-\Lambda^{3} \rho_{0}^{\prime}\right) e^{-(1+\Lambda) / \Lambda}}{\Lambda^{3} \rho_{0}} \\
&-\frac{\left(\rho_{0}+\Lambda \rho_{0}^{\prime}\right)\left(\beta-\Lambda \rho_{0}\right) e^{-(1+\Lambda) / \Lambda}}{\Lambda^{2} \rho_{0}^{2}}
\end{aligned}
$$

Equating Eq. (26) to 0 gives the relationship between the optimal individual expected search time $1 / \Lambda^{*}$ for a given population boldness $b$, as presented in Fig. 6 .

\section{Intermediate population boldness $b$}

When considering the optimal foraging rate for an individual with a population of boldness $b$, we define the intermediate population boldness as the population where the intermediate individual, with expected search time $1 / \Lambda=1 / c_{2}$, is most successful, i.e when

$b=\frac{r_{2} \Lambda}{r_{1} c_{1}}$

where

$$
\begin{aligned}
r_{1}= & \frac{\Lambda\left(1-e^{-\tau_{f}^{*}}\right)+\Lambda \tau_{1}\left(\rho_{0} e^{-\tau_{f}^{*}}-1\right)}{\Lambda \tau_{f}^{*}+1} \\
& -\frac{\Lambda \tau_{1}\left(\Lambda \rho_{0}\left(1-e^{-\tau_{f}^{*}}\right)-\Lambda \tau_{f}^{*}-(1+\beta)\right)}{\left(\Lambda \tau_{f}^{*}+1\right)^{2}},
\end{aligned}
$$

$$
\begin{aligned}
& r_{2}= \frac{\rho_{0}\left(1-e^{-\tau_{f}^{*}}\right)+\Lambda \tau_{2}\left(\rho_{0} e^{-\tau_{f}^{*}}-1\right)-\tau_{f}^{*}}{\Lambda \tau_{f}^{*}+1} \\
&-\frac{\left(\tau_{f}^{*}+\Lambda \tau_{2}\right)\left(\Lambda \rho_{0}\left(1-e^{-\tau_{f}^{*}}\right)-\Lambda \tau_{f}^{*}-(1+\beta)\right)}{\left(\Lambda \tau_{f}^{*}+1\right)^{2}} \\
& \tau_{1}=-\frac{z_{1} W(z)}{z(1+W(z))}
\end{aligned}
$$




$$
\begin{aligned}
& \tau_{2}=-\frac{z_{2} W(z)}{z(1+W(z))}+\frac{1}{\Lambda^{2}}, \\
& z_{1}=-\frac{\beta e^{-(1+\Lambda) / \Lambda}}{\Lambda \rho_{0}^{2}} \\
& z_{2}=\frac{\left(\beta-\beta \Lambda-\Lambda \rho_{0}\right) e^{-(1+\Lambda) / \Lambda}}{\Lambda^{3} \rho_{0}} .
\end{aligned}
$$

\section{Varying the shape of the trade-off curve for optimal rate analysis}

How does the shape of the trade-off curve in Eq. (17) affect these results? By varying parameters $c_{1}$ and $c_{2}$, we alter the shape of the curve and show the effects of changing initial patch qualities and expected search times for the whole population. Increasing $c_{1}$ increases $\rho_{0}$ by a factor of $c_{1}$ for all individuals on the trade-off curve, which increases an individual's patch quality. Increasing $c_{2}$ increases $\Lambda$ by a factor of $c_{2}$ for all individuals on the trade-off curve, which decreases an individual's expected search time.

When varying $c_{2}$, an individual's position on the tradeoff curve is now defined by $c_{2} / \Lambda$, as this maintains their position on the trade-off curve as the shape of the curve is altered, and so too is their expected search time.

Supplementary Figure S1 shows the effects of varying $c_{1}$ and $c_{2}$ on the optimal individual within a population of boldness $b$. As we increase patch quality by increasing $c_{1}$, we see the optimal expected search time within the population increase, so the optimal individual foraging strategy becomes more shy. If we decrease expected search times by increasing $c_{2}$, this also favours shy individuals, with the optimal individual strategy within the population favouring individuals with longer expected search times.

\section{Derivation of minimal risk individual in a population}

Let

$P^{c}=\mathrm{P}\left(\mu_{s}>\mu_{f}^{c}\right)=\left(\rho_{0} e^{-\left(\rho_{0}-1\right)}\right)^{\Lambda /(1+\beta)}$

as in Eq. (21). Differentiating $P^{c}$ with respect to $\Lambda$ gives

$\frac{d P^{c}}{d \Lambda}=-\frac{1}{1+\beta}\left(\rho_{0}+\Lambda \rho_{0}^{\prime}-\tau_{f}^{c}-\Lambda \tau_{f}^{c^{\prime}}-1\right) P^{c}$,

where $\rho_{0}$ is the foraging strategy trade-off as defined in Eq. (17), $\rho_{0}^{\prime}$ is its derivative with respect to $\Lambda$ as defined in Eq.

(27), $\tau_{f}^{c}$ is the minimal risk strategy as defined in Eq. (20) and $\tau_{f}^{c^{\prime}}=\frac{d \tau_{f}^{c}}{d \Lambda}=\frac{\rho_{0}^{\prime}}{\rho_{0}}$.

We use Eq. (38) to find the individual expected search time $1 / \Lambda$ which minimises the individual risk $P^{c}$ in a population of boldness $b$. We obtain the expression

$(b-1)\left(c_{1} c_{2}^{b} \Lambda^{-b}\right)^{2}-c_{1} c_{2}^{b} \Lambda^{-b}+$
$\left(c_{1} c_{2}^{b} \Lambda^{-b}+1\right) \ln \left(c_{1} c_{2}^{b} \Lambda^{-b}+1\right)=0$,

which gives the minimal risk expected search time $1 / \Lambda_{c}$ for an individual in a population of boldness $b$.

\section{Intermediate population boldness $\boldsymbol{b}$}

When considering the minimal risk of foraging unsuccessfully for an individual with a population of boldness $b$, we define the intermediate population boldness as the population where the intermediate individual, with expected search time $1 / \Lambda=1 / c_{2}$, is least likely to be unsuccessful, i.e when

$b=\frac{\left(c_{1}+1\right)\left(c_{1}-\log \left(c_{1}+1\right)\right)}{c_{1}^{2}}$.

\section{Varying the shape of the trade-off curve for minimal risk analysis}

We again consider the effects of changing the shape of the trade-off curve in Eq. (17), defining individual foraging strategies within the population on the curve by $c_{2} / \Lambda$. In Supplementary Figure S2, as we increase $c_{1}$, we increase the patch quality for individuals, which causes the minimal risk individual strategy to become more bold. However, as we vary $c_{2}$, varying expected search times for the population as a whole, the minimal risk individual foraging strategy is unaffected. We still see variation in the risk to each individual $P\left(\mu_{s}>\mu_{f}\right)$ as in Fig. 3 , but the individual with the lowest risk remains unchanged.

Supplementary information The online version contains supplementary material available at https://doi.org/10.1007/s12080-021-00517-7.

Acknowledgements PMJ acknowledges funding from the Natural Environment Research Council (NERC) via the 'Adapting to the Challenges of a Changing Environment' (ACCE) Doctoral Training Partnership. We thank Stephanie Peacock, an associate editor, and an anonymous reviewer for comments that have helped improve the manuscript.

Author contributions JRP and SMP conceived the research idea. JRP, SMP, PMJ designed the research. PMJ performed the research. PMJ led the writing of the manuscript; all authors contributed critically to the drafts and gave final approval for publication. 
Funding PMJ was funded by a Doctoral Training Programme from the Natural Environment Research Council (NERC).

\section{Declarations}

Consent for publication All authors have consented to publication.

Open Access This article is licensed under a Creative Commons Attribution 4.0 International License, which permits use, sharing, adaptation, distribution and reproduction in any medium or format, as long as you give appropriate credit to the original author(s) and the source, provide a link to the Creative Commons licence, and indicate if changes were made. The images or other third party material in this article are included in the article's Creative Commons licence, unless indicated otherwise in a credit line to the material. If material is not included in the article's Creative Commons licence and your intended use is not permitted by statutory regulation or exceeds the permitted use, you will need to obtain permission directly from the copyright holder. To view a copy of this licence, visit http://creativecommons.org/licenses/by/4.0/.

\section{References}

Bolnick DI, Svanbäck R, Fordyce JA, Yang LH, Davis JM, Hulsey CD, Forister ML (2003) The ecology of individuals: incidence and implications of individual specialization. Am Nat 161(1):1-28

Charnov EL (1976) Optimal foraging, the marginal value theorem. Theor Popul Biol 9(2):129-36

Dingemanse NJ, Both C, Drent PJ, Tinbergen JM (2004) Fitness consequences of avian personalities in a fluctuating environment. Proc R Soc London Ser B 271(1541):847-852

Dunn RE, White CR, Green JA (2018) A model to estimate seabird field metabolic rates. Biol Lett 14(6):20180190

Eliassen S, Jørgensen C, Mangel M, Giske J (2007) Exploration or exploitation: life expectancy changes the value of learning in foraging strategies. Oikos 116(3):513-523

Elliott KH, Chivers LS, Bessey L, Gaston AJ, Hatch SA, Kato A, Osborne O, Ropert-Coudert Y, Speakman JR, Hare JF (2014) Windscapes shape seabird instantaneous energy costs but adult behavior buffers impact on offspring. Mov Ecol 2(1):17

Furness RW, Bryant DM (1996) Effect of wind on field metabolic rates of breeding northern fulmars. Ecology 77(4):1181-1188
Furness RW, Tasker ML (2000) Seabird-fishery interactions: quantifying the sensitivity of seabirds to reductions in sandeel abundance, and identification of key areas for sensitive seabirds in the north sea. Mar Ecol Prog Ser 202:253-264

Gabrielsen GW, Mehlum F, Nagy KA (1987) Daily energy expenditure and energy utilization of free-ranging black-legged kittiwakes. The Condor 89(1):126-132

Higginson AD, Ruxton GD (2015) Foraging mode switching: the importance of prey distribution and foraging currency. Anim Behav 105:121-137

Laguë M, Tania N, Heath J, Edelstein-Keshet L (2012) The effects of facilitation and competition on group foraging in patches. $\mathrm{J}$ Theor Biol 310:88-96

Nonaka E, Holme P (2007) Agent-based model approach to optimal foraging in heterogeneous landscapes: effects of patch clumpiness. Ecography 30(6):777-788

Oaten A (1977) Optimal foraging in patches: a case for stochasticity. Theor Popul Biol 12(3):263-285

Patrick SC, Weimerskirch H (2014) Personality, foraging and fitness consequences in a long lived seabird. PloS One 9(2):e87269

Patrick SC, Charmantier A, Weimerskirch H (2013) Differences in boldness are repeatable and heritable in a long-lived marine predator. Ecol Evol 3(13):4291-4299

Patrick SC, Pinaud D, Weimerskirch H (2017) Boldness predicts an individual's position along an exploration-exploitation foraging trade-off. J Anim Ecol 86(5):1257-1268

Rodríguez-Gironés MA, Santamaría L (2006) Models of optimal foraging and resource partitioning: deep corollas for long tongues. Behav Ecol 17(6):905-910

Rueffler C, Van Dooren TJ, Metz JA (2006) The evolution of resource specialization through frequency-dependent and frequency-independent mechanisms. Am Nat 167(1):81-93

Sih A, Bell AM, Johnson JC, Ziemba RE (2004) Behavioral syndromes: an integrative overview. Q Rev Biol 79(3):241-277

Weimerskirch H (2007) Are seabirds foraging for unpredictable resources? Deep-Sea Res II Top Stud Oceanogr 54(3-4):211-223

Weimerskirch H, Guionnet T, Martin J, Shaffer SA, Costa D (2000) Fast and fuel efficient? Optimal use of wind by flying albatrosses. Proc R Soc London Ser B 267(1455):1869-1874

Wolf M, McNamara JM (2012) On the evolution of personalities via frequency-dependent selection. Am Nat 179(6):679-692

Wolf M, Van Doorn GS, Leimar O, Weissing FJ (2007) Life-history trade-offs favour the evolution of animal personalities. Nature 447(7144):581-584 